\title{
Gateway cloning and in-planta transformation of drought stress responsive Ecmyb1 gene isolated from Eleusine coracana var.PRM 6107
}

\author{
Bhatt, M. ${ }^{1}$ 凶, Salvi, P. ${ }^{2}$, Lohani, . $^{1}$
}

\begin{abstract}
Drought is one of the key abiotic stress that critically influences the crops by restraining their growth and yield potential. Being sessile, plants tackle the detrimental effects of drought stress via modulating the cellular state by changing the gene expression. Such alteration of gene expression is essentially driven by the transcriptional syndicate. Transcription factors (TF) are the key regulatory protein that controls the expression of their target gene by binding to the cis-regulatory elements present in the promoter region. Myb-TFs ubiquitously present in all eukaryotes belong to one of the largest TF family, and play wide array of biological functions in plants including anthocyanin biosynthesis, vasculature system, cell signaling, seed maturation and abiotc stress responses. In the present study the full length Myb TF from Eleusine corocana was subcloned using Gateway cloning system and further transformed into Arabidopsis thaliana through floral dip method. Transgenic Arabidopsis thaliana plants harbouring Ecmybl gene were screened and grown in transgenic glasshouse under controlled conditions.
\end{abstract}

Key words: Abiotic stress, Transcriptional regulation, MY-transcription factor, Gateway cloning, Finger millet

\section{Introduction}

Global change in climatic conditions, population and environmental degradation has led to extreme depletion of water resources thus causing widespread drought. Greenhouse emission rise has led to erratic rainfall, increase in parched land area, desertification and ultimately reduction in crop productivity. Drought is a major abiotic stress that is a cause of severe agony to humankind. It is generally defined as protracted time period of scarce precipitation compared to the statistical cumulative average for a region that results in water shortage for some activity, group or environmental (Leng and Hall, 2019, Jie et al, 2020, southasiadisasters.net March 2015). It is a major abiotic stress that culminates in extreme loss of yield to crops and has proven to be more scourging than any other abiotic hazard. Since 1900, a causality of more than 11 million people has been logged and approximately 2 billion have been influenced by drought conditions (Boyer, 2013). In

\section{Author's Address}

${ }^{1}$ Department of Molecular Biology and genetic Engineering, CBSH, GBPUniversity of Ag. \& Tech. Pantnagar, India

${ }^{2}$ Agriculture Biotechnology Department National Agriculture Biotechnology Institute, Mohali, Punjab, India

E-mail.: megha4bhatt@gmail.com order to overcome such problem an important approach is to generate crops with enhanced drought tolerance, by utilizing biotechnology and molecular biology techniques. This strategy requires the identification of traits relating to drought tolerance and then the genes that determine these traits. Plants often carry genes that enable them to maintain their performance despite severe moisture stress conditions. Such plants are referred to as drought tolerant and for tolerance several genes have been recognized and characterized.

Further, it is necessary to explore the molecular and genetic bases behind drought tolerance and genes that are responsible for coding the major transcription factor and their functional proteins (Kapoor, et al., 2020). These stress-inducible genes can be classified into two groups. The first group includes proteins that function in abiotic stress tolerance.

These constitute of molecules such as late embryogenesis abundant (LEA) proteins, chaperones, antifreeze proteins, mRNA-binding proteins, osmotin, key enzymes for osmolyte biosynthesis, sugar, water channel proteins and proline transporters, detoxification enzymes and various proteases. The second group comprises of 
regulatory proteins. These include various transcription factors, protein kinases, protein phosphatases, enzymes involved in phospholipid metabolism, and other signaling molecules (Ciarmiello and Woodrow, 2011).Thus, drought tolerance is a multigenic quantitative trait involving complex genetic control.

Till date researchers have not been completely successful in developing drought tolerant cultivars by targeting a single gene (Kaur et al., 2015). Therefore, research on the master regulators that modulate expression of all such genes gained limelight. The master regulators are the transcription factors that are induced at the onset of drought conditions (Salvi et al., 2016). They bind specifically to the promoters of downstream genes associated with drought tolerance and upregulate their expression. For abiotic stress response mechanism various transcription factors have been known so far (Negi et al., 2017). An important transcription factor amongst these is the MYB transcription factor. The MYB proteins are characterized by a MYB DNA binding domain in their N-terminus that is composed of one or more imperfect tandem repeat(s).

MYB with the R2R3 type repeat is found in plants and have been reported to be responsible for influencing the expression of $\mathrm{ABA}$ regulated drought tolerance related genes (Saxena et al., 2013, 2020). The expression of MYB transcription factor gene has been studied in finger millet. The expression of the myb gene is conditional and takes place only with the onset of drought (Salvi et al., 2012). The expression level increases many folds with the increase in the severity of drought (Jadhav et. al., 2018).

Finger millet a member of poeceae, is a hardy crop and shows tolerance towards abiotic stresses specifically drought. Finger millet can survive in harsh environmental stress conditions due to their potent alleles which show drought resistant characteristics. Such crops will be of great significance in meeting challenges due to water shortage (Gull et al., 2014). Therefore, in present investigation cloned full length sequence of drought stress inducible Ecmybl gene was first subcloned through Gateway vector system and further transformed into Arabidopasis thaliana through floral dip method.
Materials and Methods

Isolation and cloning of full-length gene encoding Ecmyb1

In our previous study we have identified and isolated a partial gene (CDS) encoding MYB-TF from Eleusine coracana which was found to induced after drought stress exposure. To further understand the molecular role and regulation of the Ecmyb1, we sought out to clone full length sequence of the gene. In order to clone the fulllength gene, we isolated the RNA from the Eleusine coracana leaves and reverse transcribed it to cDNA. The cDNA was used to amplify the fulllength gene amplicon of Ecmybl, and subsequently cloned in pGEM-T vector system according to manual instruction. The cloned amplicon was confirmed by sequencing and subsequently submitted to NCBI GenBank.

Primers used for full length sequence were 5'TCAACTAATGGTAGCCCTTCCCTCT-3' (sense) and 5'- GATATTCTCAAAAGACAGTTGCATTCT3' (antisense). For designing the primer sequences, partial sequence of Ecmyb1 gene which has been submitted with Genbank accession number JN107890 was nBLAST with Eleusine coracana whole genome sequence (GenBank: LXGH01099917.1).The sequence that showed similarity was considered for ORF searched using ORF finder tool (NCBI). The lateral flanking sequence of ORF was used for primer designing.

\section{Gateway cloning of full length Ecmyb1 gene for functional analysis and expression analysis}

The Gateway Technology utilizes the site specific recombination system of lambda. The principle behind this process is to enable the incorporation of lambda genome into E.coli chromosome and consequently this integration leads to transition between lytic and lysogenic cycle.For Gateway system first step is to generate an entry clone in which our gene of interest will be present and is flanked by modified att sites. Entry clone was generated using TOPO cloning method as described in manual (Ptashne, 1992). Single colony was taken from positive selected transformed cells after colony PCR selection and PCR was carried out using primers sense (5'CACCATGGTAGCCCTTCCCTCTGCGATG3') and antisense (5'GATATTCTCAAAAGACAGTTGC3'). These primers were same as gene specific primers with 4 base pair sequence CACC which was 
necessary for directional cloning on the $5^{\prime}$ end of the forward primer.Further transformation was carried out using competent E. coli cell. Colony PCR was performed to check the trans-formants of the desired fragments. The recombination reaction of an attLsubstrate is indicated as entry clone which fuses with an att $\mathrm{R}$ substrate known as destination vector. This reaction generates an attB containing the expression clone in which our gene of interest will be present along with the by product containing attP sequence. This process is known as LR reaction. Single positive colony was taken from the bacterial plate containing Ecmyb1 gene: E.coli DH5 $\alpha$ : TOPO vector (entry clone) and PCR reaction was carried out using M13 forward and M13 reverse primers sense (5'GTAAAACGACGGCCAGT 3') and antisense (5'AACAGCTATGACCATGT 3'). Further, colony-PCR was carried out to check the transformants of the desired fragments using Ecmybl gene specific primers.

\section{Agrobacterium tumefaciens mediated gene transformation in Ecmyb1 gene}

Primary culture was prepared by inoculating a single colony of Agrobacterium tumefaciens strain EHA105 in $5 \mathrm{ml}$ of LB medium with rifampicin $(10 \mathrm{mg} / \mathrm{l})$ and incubated at $28^{\circ} \mathrm{C}$ with overnight shaking at $200 \mathrm{rpm}$. The primary culture $(2 \mathrm{ml})$ was transferred to $50 \mathrm{ml} \mathrm{LB}$ broth containing rifampicin $(10 \mathrm{mg} / \mathrm{l})$ antibiotic and grown till an optical density of 0.5 is achieved. Cells were pelleted by centrifugation at $5,000 \mathrm{~g}$ for 5 minutes at $4^{\circ} \mathrm{C}$ and supernatant was discarded. The pellet was resuspended in $10 \mathrm{ml}$ of pre-chilled $0.5 \mathrm{M} \mathrm{NaCl}$ and was again centrifuged at $5,000 \mathrm{~g}$ for 5 minutes at $4^{\circ} \mathrm{C}$. Finally, the pellet was resuspended in $1.0 \mathrm{ml}$ of ice-cold $20 \mathrm{mM} \mathrm{CaCl}_{2}$ and transformed by freeze thaw method.

\section{Showing of Arabidopsis thaliana ecotype Columbia seeds}

Seeds were washed properly and kept in dark at $4^{0}$ $\mathrm{C}$ for stratification process for 2-3 days. Seeds were then inoculated in Jam bottles containing half strength of MS media for germination. After ten to fifteen day seedlings were transferred to pots that contained autoclaved soil (soil vermiculite in ratio of 3:10). Arabidopsis plants were grown till flowering stage in a greenhouse with temperatures $24^{\circ} \mathrm{C}$ day $/ 20^{\circ} \mathrm{C}$ night.

\section{Floral Dip Method for gene transformation}

Positive single colony was taken from the bacterial plate generated after transformation with Agrobacterium (EHA105:pMDC84:Ecmyb1) and inoculated in YEP media for 48 hours at $28^{\circ} \mathrm{C}$ with $200 \mathrm{rpm}$ in a shaker in the presence of antibiotic rifampicin $(10 \mathrm{mg} / \mathrm{l})$ and kanamycin $(50 \mathrm{mg} / \mathrm{l})$. After 48 hours when the $\mathrm{OD}_{600}$ of the suspension culture reached to 0.8 , culture was then centrifuged at $4000 \mathrm{~g}$ for 10 minutes at room temperature. Harvested cells were gently re-suspended in $250 \mathrm{ml}$ of freshly made $5 \%(\mathrm{w} / \mathrm{v})$ sucrose solution. Surfactant Silwet L-77 was added to this solution at a concentration of $0.02 \% \quad(\mathrm{v} / \mathrm{v})$ and stirred uniformly. The aerial parts of the plant (at 50\% flowers open stage) were dipped in this solution for about 5-10 seconds, with gentle agitation. The pots containing inoculated plants were covered with perforated plastic polybag for 16-24 hours to maintain high humidity. The treated plants were allowed to grow to full maturity. After the plants reached complete maturity and dried, the seeds were carefully harvested and screened for transformants.

\section{Screening of primary transformants}

For screening of primary transformants, $\mathrm{T}_{0}$ seedling of Arabidopsis thaliana was used. Seeds were surface sterilized by treating them with $70 \%$ ethanol for $1 \mathrm{~min}$ followed by treatment with $1 \%$ bleach solution for 1-2 min. Seed were then rinsed three times with sterile water till the water appeared clear without any yellow color. Presence of yellow color indicates that residual bleach is still present in the wash. Sterilized seeds were kept for stratification process of 2-3 days. Seeds were then inoculated in jam bottles containing half strength of MS media with antibiotic hygromycin $25 \mathrm{mg} / 1$ for selection of transformants and placed in a tissue culture room under continuous light (21500 lux photon) conditions. Transformed plantlets appeared from the seeds after $7-10$ days.

\section{PCR confirmation of transformed seedlings of Arabidopsis thaliana using hygromycin primers} The positively selected transformed plants were transferred from MS medium to soil under shaded 
green house until flowering stage. Further, these seedlings were used for DNA isolation and PCR reaction was executed using hygromycin primers sense (5'TTCAGCTTCGATGTAGGAGGG3') and antisense (5'ATGTTGGCGACCTCGTATTGG 3').

\section{Results and Discussion}

Full length Ecmybl gene was amplified using gene the specific primers. After PCR the product was cloned in pGEM-T easy vector and further sequenced. The Ecmybl gene contained 1053 nucleotides that were translated to 350 amino acid sequence using EXPASY tool (Fig 1). Complete sequence of Ecmybl gene was submitted to NCBI with accession number MT312253. Additionally, $E c m y b 1$ gene was subcloned into plant expression vector pMDC84 using Gateway system. The confirmed cloned were transformed in Agrobacterium tumefaciens strain EHA105.

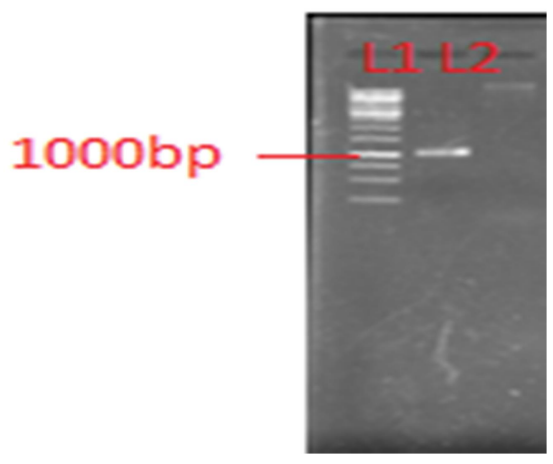

Figure 1: L1-1 kb plus DNA Ladder, L-2 1053 bp fragment of Ecmyb1 gene after colony PCR

Transformation of EcMyb1 gene into Arabidopsis thaliana through floral dip method In-planta transformation protocol in Arabidopsis thaliana and others plant systems through Agrobacterium mediated method has made many successful attempts (Zia et al., 2011). In Arabidopsis thaliana transformation through Inplanta methods early success was achieved by the Feldmann et al., 1987 work. Later Bechtold et al., (1993) reported that in Arabidopsis thaliana the transformation rate enhanced if plants were inoculated at flowering stage(Supartana et al., 2006). In floral dip method Agrobacterium containing Ecmyb1 gene (Fig 2 ) was grown in YEP medium supplemented with antibiotic (kanamycin and hygromycin) at $28^{\circ} \mathrm{C}, 250 \mathrm{rpm}$ for 48 hours(TianZi et al., 2010). After 48 hours cells were harvested by centrifugation and suspended in $250 \mathrm{ml}$ of freshly made $5 \%(\mathrm{w} / \mathrm{v})$ sucrose and 0.02 $\%$ solution of surfactant Silwet L-77. In the next step aerial parts of Arabidopsis plants were inverted and gently stirred in the suspension for 5to $10 \mathrm{sec}$. Sucrose and silvet L-77 are very important for Agrobacterium infection. Sucrose helps in activation of vir genes by providing a signal whereas Silwet L-77 increases the entry of Agrobacterium by reducing the surface tension (Clough et al., 2000).

Subsequently, pots were covered with plastic bag to create humidity for maintaining adequate environment for Agrobacterium infection. Arabidopsis thaliana plants were grown for transformation until maturity after which seeds were collected and used for selection of Ecmybl gene.

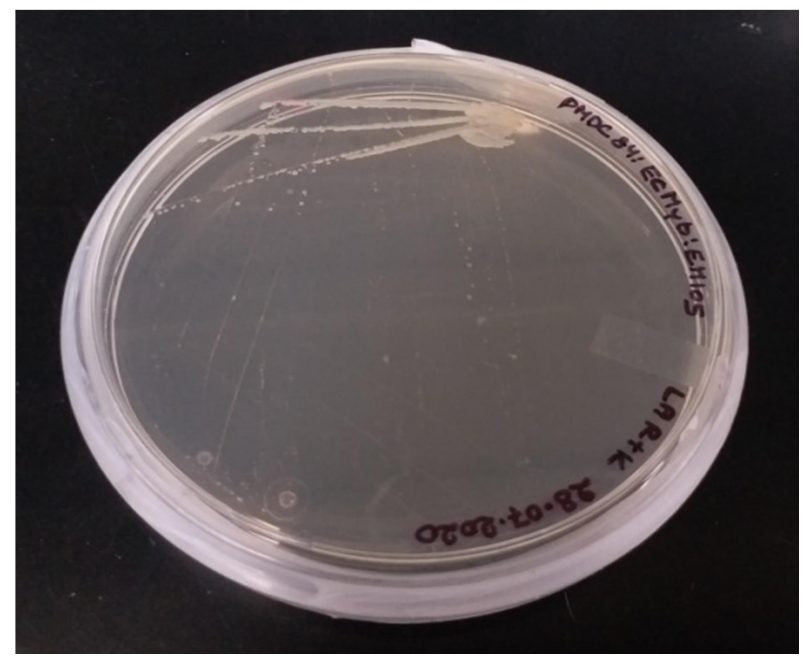

Figure 2: Transformed colonies of PMDC84 : Ecmyb1 gene Selection was carried out by using kanamycin $(25 \mathrm{mg} / \mathrm{L})$ and rifampicin $(10 \mathrm{mg} / \mathrm{l})$

\section{Hygromycin based selection of transgenic} Arabidopsis thaliana plants

The Agrobacterium infected Arabidopsis thaliana plants were further selected on hygromycin. For hygromycin selection seeds were grown on half strength of MS media with $25 \mathrm{mg} / 1$ antibiotic hygromycin. Only transformed seeds were able to 
grow on hygromycin medium (Fig3). The possible reason behind this selection is hygromycin which interferes with protein synthesis process of non transformed cells. Hygromycin competes with the binding site between elongation factor FE-2 and ribosome. This interference destroys the function of ribosome which ultimately leads to inhibition in protein synthesis process and makes plants sensitive to hygromycin (Duan et al., 2011).

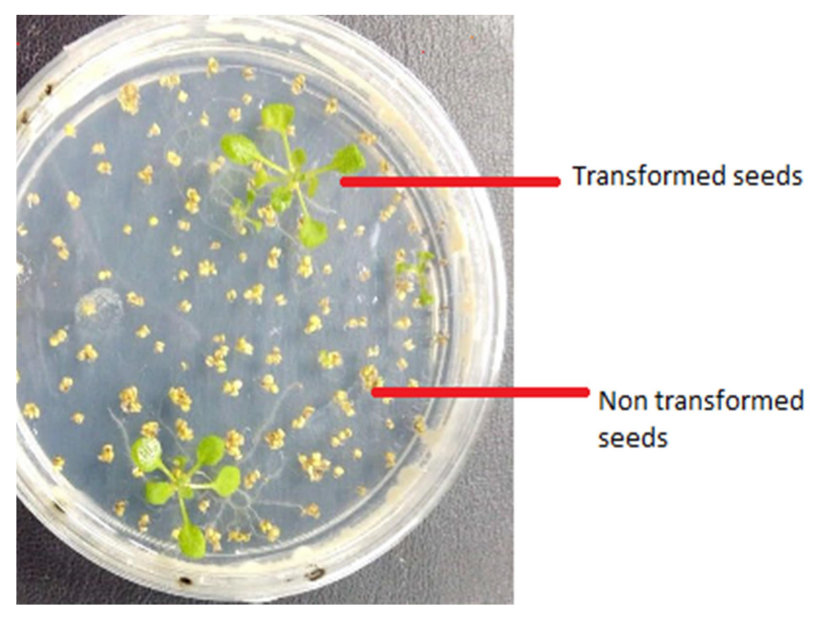

Figure 3: Tansgenic seedlings transformed with pMDC84:Ecmyb1 gene selected on $25 \mathrm{mg} / \mathrm{l}$ hygromycin, non transformed seeds were unable to grow in hygromycin.

PCR confirmation of transformed seedlings of Arabidopsis thaliana using hygromycin primers Seedlings of Arabidopsis thaliana which was selected through hygromycin selection were further processed for PCR to confirm the T-DNA inserted containing Ecmybl gene. The positively selected transformed plants were transferred from MS medium to soil under shaded green house until flowering stage. Further, genomic DNA was isolated from these seedlings and PCR reaction was performed using hygromycin primers. PCR generates amplicon size of $587 \mathrm{bp}$ that corresponds to size of hygromycin sequence present in vector (Fig 4). Transformation efficiency for floral dip method in this study was $1 \%$ as out of 700 seeds 7 transgenic plants were screened in hygromycin selection (Fig 4). This result obtained in the present study were in the acceptable range $(0.5-3 \%)$ as established by the study of Clough and Bent 1998; 2000).

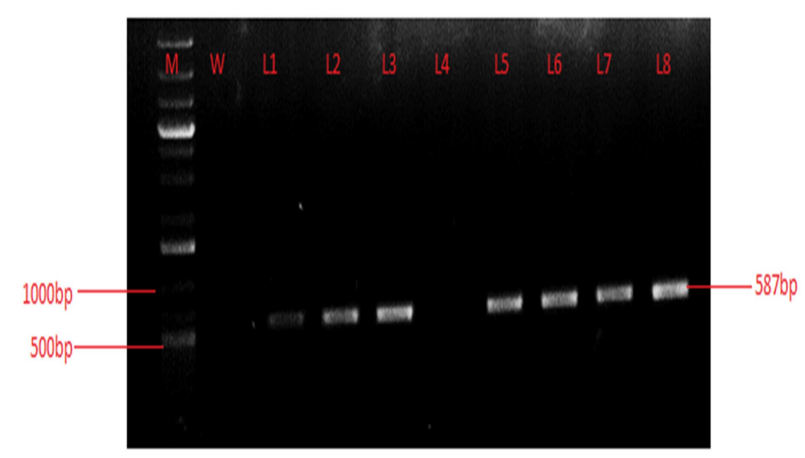

Figure 4: PCR confirmation of transgenic Arabidosis thaliana seedlings containing Ecmyb1 gene with primer specific to hygromycin gene using genomic DNA as template. L- 1000bp ladder, W-wild type Arabidopsis plants (no band), L- 1,2,3,5,6,7 transformed plants showing amplicon size of $587 \mathrm{bp}$

\section{Conclusion}

Drought is a foremost abiotic stresses that harmfully influences the crops by restraining their growth and immensely reducing the yield capacity. Henceforth, it is necessary to comprehend the mechanisms that support plants to handle the deficiency of water. Plants often carry drought tolerance genes that enable them to maintain their performance despite of severe moisture stress conditions, such plants are referred to as drought tolerant plants. Transcription factors (TF) are mainly responsible for creating molecular level changes in plants during drought stress condition. They build strong association with general transcription factor at promoter of target gene and regulate their expression. Association of transcription factors with each other developed in response to intracellular signals which leads to activation or repression of target gene. An important transcription factor amongst these is the MYB transcription factor. The specialized characteristic of MYB protein is that they contain MYB DNA binding domain in their N-terminus which is composed of one or more imperfect tandem repeat(s). In this study, we have subcloned a full-length coding sequence of a drought responsive gene that encodes MYB- transcription factor which was already submitted to NCBI (Accession number MT312253) through Gateway system. Transgenic Arabidopsis thaliana plants harbouring Ecmyb1 gene were produced in the 


\section{Bhatt et al.}

present study. The transgenic plants will be maintained and screened for two generations to attain homozygosity. After functional validation study of the gene in Arabidopsis, it could be recommended for transfer in other plants like rice or maize to produce drought tolerant crops.

\section{References}

Bechtold, N., 1993. In planta Agrobacterium-mediated gene transfer by infiltration of adult Arabidopsis plants. $\boldsymbol{C R}$ Academic Science Series, III Sci. View.316:1194-1199.

Bent, A.F. and Clough, S.J., 1998. Agrobacterium germ-line transformation: transformation of Arabidopsis without tissue culture. In Plant molecular biology manual Springer, 17-30.

Bent, A.F., 2000. Arabidopsis in planta transformation. Uses, mechanisms, and prospects for transformation of other species. Plant physiology, 124(4):1540-1547.

Boyer, J. S., Byrne, P., Cassman, K. G., Cooper, M., Delmer, D., Greene, T., Gruis, F., Habben, J., Hausmann, N., Kenny, N. and Lafitte, R. 2013. The US drought of 2012 in perspective: A call to action. Global Food Security, 2(3): $139-143$

Ciarmiello, L. F., Woodrow, P., Fuggi, A., Pontecorvo, G. and Carillo, P. 2011. Plant genes for abiotic stress. Abiotic stress in Plants-Mechanisms and Adaptations, 283-308.

Clough, S. J., Fengler, K. A., Yu, I. C., Lippok, B., Smith, R. K. and Bent, A. F. 2000. The Arabidopsis dnd1 "defense, no death" gene encodes a mutated cyclic nucleotide-gated ion channel. Proceedings of the National Academy of Sciences, 97(16): 9323-9328.

Clough, S.J. and Bent, A.F., 1998. Floral dip: a simplified method for Agrobacterium-mediated transformation of Arabidopsis thaliana. The Plant Journal, 16(6): 735-743.

Duan, H., Ding, X., Wei, Z., Zhou, C. and Zhou, Y. 2011. The influences of Hygromycin B on growth of Arabidopsis thaliana cotyledon and leaf. African Journal of Biotechnology, 10(77): 17742-17747.

Feldmann, K.A. and Marks, M.D., 1987. Agrobacteriummediated transformation of germinating seeds of Arabidopsis thaliana: a non-tissue culture approach. Molecular and General Genetics, 208(1-2): 1-9.

Gull, A., Jan, R., Nayik, G. A., Prasad, K. and Kumar, P. 2014.Significance of finger millet in nutrition, health and value added products: a review. Journal of Environment Science and Computer Science Engineering and Technology, 3: 1601-1608.

Jadhav, P., Salvi, P., Bhatt, M. and Lohani, P., 2018. Expression of ECMYB Transcription Factor Gene Under
Different Abiotic Stress Conditions in Eleusine coracana. International Journal of Agriculture, Environment and Biotechnology, 11(5): 799-806.

Jie, Z.O.U., Wei, H.U., Li, Y.X., He, J.Q., Zhu, H.H. and Zhou, Z.G., 2020. Screening of drought resistance indices and evaluation of drought resistance in cotton (Gossypium hirsutum L.). Journal of Integrative Agriculture, 19(2): 495-508.

Kapoor, D., Bhardwaj, S., Landi, M., Sharma, A., Ramakrishnan, M. and Sharma, A., 2020. The impact of drought in plant metabolism: how to exploit tolerance mechanisms to increase crop production. Applied Sciences, 10(16): 5692.

Kaur, H., Petla, B.P., Kamble, N.U., Singh, A., Rao, V., Salvi, P., Ghosh, S. and Majee, M. 2015. Differentially expressed seed aging responsive heat shock protein OsHSP18. 2 implicates in seed vigor, longevity and improves germination and seedling establishment under abiotic stress. Frontiers in Plant Science, 6: 713.

Leng, G. and Hall, J. 2019. Crop yield sensitivity of global major agricultural countries to droughts and the projected changes in the future. Science of the Total Environment, 654: 811-821.

Negi, B., Salvi, P., Bhatt, D., Majee, M. and Arora, S. 2017. Molecular cloning, in-silico characterization and functional validation of monodehydroascorbate reductase gene in Eleusine coracana. Plos one, 12(11): 0187793.

Ptashne, M. 1992. A genetic switch: phage and higher organisms. Cambridge, MA: Cell Press.

Salvi, P., Saxena, S. C., Petla, B. P., Kamble, N. U., Kaur, H., Verma, P., Rao, V., Ghosh, S. and Majee, M. 2016. Differentially expressed galactinol synthase (s) in chickpea are implicated in seed vigor and longevity by limiting the age induced ROS accumulation. Scientific Reports, 6: 35088

Salvi, P., Arora, A., and Lohani, P. 2012. Expression of MYB Transcription Factor in Eleusine coracana: towards making of drought tolerant plants, LAP Lambert Academic Publishing, USA.

Saxena, S. C., Salvi, P., Kamble, N. U., Joshi, P. K., Majee, M. and Arora, S. 2020. Ectopic overexpression of cytosolic ascorbate peroxidase gene (Apx1) improves salinity stress tolerance in Brassica juncea by strengthening antioxidative defense mechanism. Acta Physiologiae Plantarum, 42(4): $1-14$.

Saxena, S. C., Salvi, P., Kaur, H., Verma, P., Petla, B. P., Rao, V., Kamble, N. and Majee, M. 2013. Differentially expressed myo-inositol monophosphatase gene (CaIMP) in chickpea (Cicer arietinum L.) encodes a lithium-sensitive phosphatase enzyme with broad substrate specificity and improves seed germination and seedling growth under 
Gateway cloning and in-planta transformation

abiotic stresses. Journal of Experimental Botany, 64(18): 5623-5639.

South Asia disasters.net March 2015. Accessed on April 2020.

Supartana, P., Shimizu, T., Shioiri, H., Nogawa, M., Nozue, M. and Kojima, M. 2006. Development of simple and efficient in planta transformation method for rice (Oryza sativa L.) using Agrobacterium tumefaciens. Journal of Bioscience and Bioengineering, 100(4): 391-397.
TianZi, C., ShenJie, W., Jun, Z., WangZhen, G. and TianZhen, Z. 2010. Pistil drip following pollination: a simple in planta Agrobacterium-mediated transformation in cotton. Biotechnology Letters, 32(4): 547-555.

Zia, M., Arshad, W., Bibi, Y., Nisa, S. and Chaudhary, M. F., 2011. Does Agroinjection to soybean pods transform embryos. Plant Omics Journal, 4(7): 384-390. 